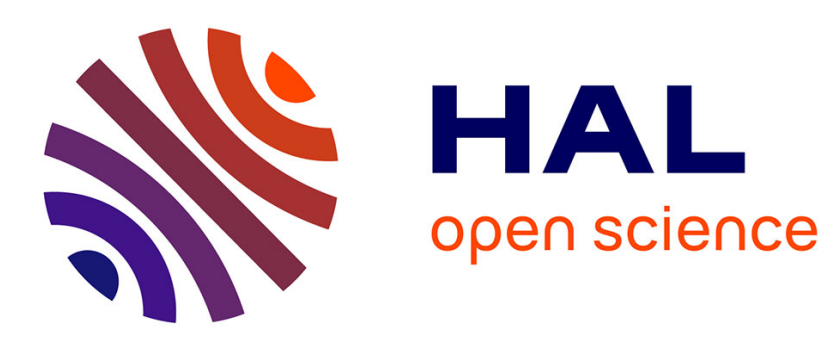

\title{
Max-plus-linear model-based predictive control for constrained HVLV manufacturing systems
}

Imed Nasri, Georges Habchi, Reda Boukezzoula

\section{To cite this version:}

Imed Nasri, Georges Habchi, Reda Boukezzoula. Max-plus-linear model-based predictive control for constrained HVLV manufacturing systems. ETFA'2011, Sep 2011, Toulouse, France. pp.1-4. hal00627566

\section{HAL Id: hal-00627566 \\ https://hal.univ-smb.fr/hal-00627566}

Submitted on 29 Sep 2011

HAL is a multi-disciplinary open access archive for the deposit and dissemination of scientific research documents, whether they are published or not. The documents may come from teaching and research institutions in France or abroad, or from public or private research centers.
L'archive ouverte pluridisciplinaire HAL, est destinée au dépôt et à la diffusion de documents scientifiques de niveau recherche, publiés ou non, émanant des établissements d'enseignement et de recherche français ou étrangers, des laboratoires publics ou privés. 


\section{Max-plus-linear model-based predictive control for constrained HVLV manufacturing systems}

\author{
Imed Nasri, Georges Habchi \\ University of Savoie \\ SYMME Laboratory-Annecy \\ (imed.nasri, georges.habchi)@univ-savoie.fr
}

\author{
Reda Boukezzoula \\ University of Savoie \\ LISTIC Laboratory-Annecy \\ reda.boukezzoula@univ-savoie.fr
}

\begin{abstract}
In this paper, a max-plus-linear model predictive control strategy is proposed for High-Variety, Low-Volume (HVLV) systems. Firstly, using the (max,+) algebra, a direct generation of event-timing equations for deterministic and decision-free HVLV manufacturing systems is obtained. Then, a linear optimization method is presented. It is based on canonical forms for Max-Min-Plus-Scaling (MMPS) functions with linear constraints on the inputs. The approach aims at solving several linear programming problems and its validity is illustrated by a simulation example. Finally, a discussion of results, conclusions and perspectives are given.
\end{abstract}

\section{Introduction}

High-Variety, Low-Volume (HVLV) manufacturing systems are a class of dynamical systems where the behavior is similar to Discrete Event Dynamic Systems (DEDS). They are characterized by a wide variety of products using shared machines, a weak and personalized demand, relatively long processing times and frequent change over and set-up times. Consequently, a continuous approximation of the production flow by continuous flow systems [ [3, [1, 团] is not appropriate. In this framework, it seems very interesting to handle this kind of systems as a Multi-Input, Multi-Output (MIMO) DEDS systems.

Production systems can be modeled from two different points of view, qualitative or quantitative. The work presented in this paper is a part of quantitative modeling. The main goal is to propose an analytical model and develop an approach able to generate the event-timing equations without the using of Petri Nets. These equations are elaborated from the system configuration for a repetitive decision-free HVLV system. A system where every machine repeats its operations in the same order during each of its cycles [ [D] with predetermined and fixed sequences of operations []. Thereafter, a Model Predictive Control (MPC) is applied to our system with the objective of meeting due dates just-in-time (JIT) production criteria.

MPC is applicable to multi-input multi-output systems, it can handle constraints, states and outputs in a systematic way and it is capable of tracking pre-scheduled reference signals.

In this paper a Max-Plus-Linear (MPL)-MPC technique associated to an optimization algorithm is applied to compute a MPC controller for a simple HVLV system. The MPL-MPC controller takes into account the objective of meeting the due dates and a Just-In-Time (JIT) production. It is based on canonical forms for MMPS functions in the case of linear input constraints [焑]. It is not the case for [ []] where we have to solve a nonlinear, nonconvex optimization.

The remainder of this paper is organized as follows. Section 2 describes a state-space HVLV systems modeling. In section 3, the MPL-MPC problem is detailed and then applied on HVLV systems. Next, discussion and practical implementation are shown. Concluding remarks and future research directions are presented in section 5 .

\section{HVLV systems modeling}

The focus of this section is to develop an approach for generating event-timing equations from the machine interconnections for a generalized deterministic and free-decision HVLV flowshop system.

\subsection{Approach principle}

Algebraic models provide many attractive features: they aim to capture the description of the trajectories of manufacturing systems in terms of a set of operations on functions of state and/or events. This representation is similar to the one employed in Continuous Variable Dynamic Systems (CVDS) by differential equations and algebraic operations [ []$]$.

Max-plus algebra is applied to represent HVLV systems where relationships between the starting times of the operations (states of the system) require maximum and addition operators. The control variables used are the instants at them raw materials are fed as late as possible to the system.

Some assumptions concerning the scheduling policy, the loading sequences and the operation of the HVLV system are posed:

- The system is deterministic and decision-free.

- The number of jobs to be processed over the planning horizon is defined in advance.

- Each machine can perform one operation at a time.

- Routing decisions are already made and each operation is assigned to a particular machine.

- Job finishing times have to meet the given due dates.

- The time at which raw material is fed to the system must be as late as possible to satisfy a JIT production. 
Max-plus algebra is used in development of algebraic models of DEDS [四]. For all $a, b \in R \cup-\infty$ the max-plus operators $\oplus$ and $\otimes$ are defined according to the following equations:

$$
\begin{gathered}
a \oplus b=\max (a, b) \\
a \otimes b=a+b
\end{gathered}
$$

For the sake of simplicity, we consider a small scale test problem of an HVLV system (i.e., a 2-machine generalized flow shop).

\subsection{Illustrative example}

A generalized flow shop system is a flow shop system in which a job does not have to visit all the machines in the system. Certain jobs may skip certain machines.

Throughout this section, it is assumed that the scheduling policy and loading sequences are repetitive and the master production schedule concerns one product $P 1$ and one product $P 2$ by batch (MPS $=(1 P 1,1 P 2))[\square]$. For this purpose, a generalized 2-machine flow shop system is considered.

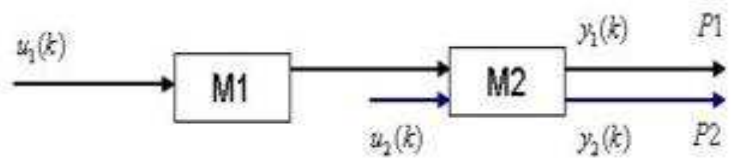

\section{Figure 1. A 2-machine generalized flow shop.}

Figure $\mathbb{U}$ shows a 2-machine HVLV flow shop. The manufacturing routing of the product $P 1$ includes two operations: the first one is processed on machine $M_{1}$ and the second one on machine $M_{2}$. The product $P 2$ is performed through a single operation on machine $M_{2}$. The objective is to derive timing equations according to the following form:

$$
\begin{gathered}
X(k)=A \otimes X(k-1) \oplus B \otimes U(k) \\
Y(k)=C \otimes X(k)
\end{gathered}
$$

Let define the following:

- $u_{i}(k)$ : time at which the raw material of the $k$ th product of type $i$ belonging to the $k$ th MPS, $i=1,2$, is fed to the system.

- $y_{i}(k)$ : time at which the $k$ th product of type $i$ belonging to the $k$ th MPS, $i=1,2$, is completed.

- $S_{l m}$ : processing time required by the machine $M_{m}, m=$ 1, 2 to process the operation $l, l=1,2,3$.

- $x_{l m}(k)$ : start time of the operation $l, l=1,2,3$ on the machine $M_{m}, m=1,2$ of the $k$ th product of type $i$ belonging to the $k$ th MPS, $i=1,2$.

- $U(k): k$ th input time vector defined as $U(k)=\left[u_{1}(k) u_{2}(k)\right]^{T}$.

- $X(k): k$ th start time vector or state vector defined as $X(k)=\left[x_{11}(k) x_{22}(k) x_{32}(k)\right]^{T}$.

- $Y(k): k$ th completion time vector or output vector defined as $Y(k)=\left[y_{1}(k) y_{2}(k)\right]^{T}$.
- $A, B$ and $C$ are adequate (max,+) constant matrices describing the relationships among different variables of the system.

Table 1. Production data

\begin{tabular}{|c|c|c|c|}
\hline Products & Operation & Machine & Processing time \\
\hline Product 1 & 1 & 1 & 1 \\
- & 2 & 2 & 2 \\
\hline Product 2 & 3 & 2 & 1 \\
\hline
\end{tabular}

Within this context, we can then proceed to develop a dioid algebraic state-space model. Let $M$ denote the set of all available machines in the system, $N$ the set of all jobs introduced to the system, and $P$ the set of all the operations to be produced on the jobs over the next planning horizon.

Let $x_{l m}(k)$ be the start time of machine $m$ for operation $l$ and for the $k$ th MPS, then the completion time of operation $l$ on machine $m$ is equal to its processing time $S_{l m}$ plus its start time $x_{l m}(k)$. Two situations may arise.

If operation $l$ is the first operation on the job, then its processing start time $x_{l m}(k)$ is determined by the maximum of either:

- the completion time of all operation, $p \in P$, on machine $m \in M$ for the $(k-1)$ th batch (MPS),

- its control variable $u_{i}(k)$.

In the second situation, if operation $l$ is not the starting operation for the job, then its processing start time $x_{l m}(k)$ is determined by the maximum of either:

- the completion time of all operation, $p \in P$, on machine $m \in M$ for the $(k-1)$ th batch (MPS),

- the completion times of all its predecessors, say $n \in P$, being processed on their respective machines, say $t \in M$, for the $k$ th MPS.

Referring to the above assumptions, the dynamics of the machines for the example considered can be now derived according to the following equations:

$$
\begin{gathered}
x_{11}(k)=\max \left(S_{11}+x_{11}(k-1), u_{1}(k)\right) \\
x_{22}(k)=\max \left(S_{22}+x_{22}(k-1), S_{32}+x_{32}(k-1),\right. \\
\left.S_{11}+x_{11}(k)\right) \\
x_{32}(k)=\max \left(S_{22}+x_{22}(k-1), S_{32}+x_{32}(k-1),\right. \\
\left.S_{22}+x_{22}(k), u_{2}(k)\right) \\
y_{1}(k)=S_{22}+x_{22}(k) \\
y_{2}(k)=S_{32}+x_{32}(k)
\end{gathered}
$$

\section{HVLV systems control}

The main contribution of this section is the application of a MPC framework based on linear optimization problem for the HVLV systems. The goal is to determine the greatest input in terms of time for the raw material to be fed in the system over the planning horizon such that the given sequences of due dates are met and JIT objectives are achieved. 


\subsection{Approach principle}

In this section, we introduce the Model Predictive Control (Figure D) used to solve a JIT tracking problem. The fundamental principles of general MPC are: Prediction, performance evolution, optimization and control action.

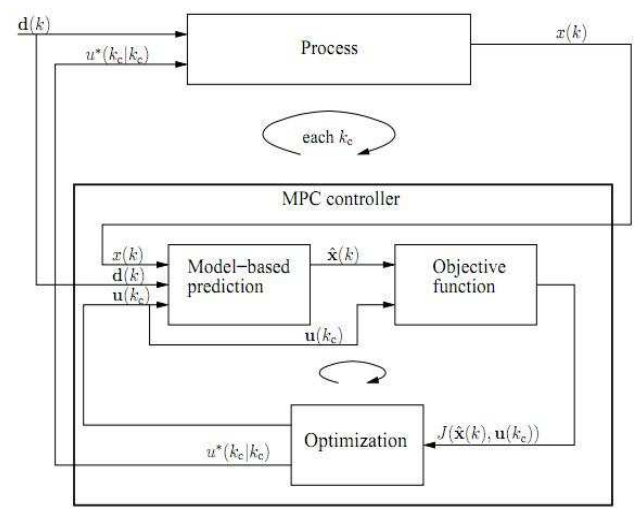

Figure 2. The full MPC scheme.

MPC [䧃] is a model-based control approach allowing to handle constraints on the inputs and outputs. It uses a receding finite horizon approach.

At the $k$ th step event the future control sequence $u(k), \ldots$, $u(k+N c-1)$, where $N c$ is the control horizon, is determined such that the cost criterion is minimized subject to the constraints. At step event $k$ the first element of the optimal sequence $(u(k))$ is applied to the process. When the next event of the horizon is shifted, the model is updated with new information, and a new optimization at the event of step $k+1$ is performed.

The cost criterion $J(k)=J_{\text {out }}(k)+\lambda J_{\text {in }}(k)$ used in MPLMPC reflects the reference tracking error $\left(J_{\text {out }}\right)$ and the control effort $\left(J_{i n}\right)$, where $\lambda$ is a nonnegative weight parameter. Let $r$ denote and define the reference signal, and define the vectors [ [ 6 ]

$$
\tilde{y}(k)=\left[\hat{y}^{T}(k \mid k) \ldots \hat{y}^{T}(k+N p-1 \mid k)\right]^{T}
$$

where $N p$ is the prediction horizon.

$$
\tilde{u}(k)=\left[u^{T}(k) \ldots u^{T}(k+N c-1)\right]^{T},
$$

and the vector of references (dues dates of products)

$$
\tilde{r}(k)=\left[r^{T}(k) \ldots r^{T}(k+N p-1)\right]^{T} .
$$

Then we have [5]:

$$
J_{\text {out }}(k)=\oplus_{j=0}^{N p-1} \oplus_{i=1}^{l}\left|\hat{y}_{i}(k+j \mid k)-r_{i}(k+j)\right|
$$

where $l$ is the number of outputs.

$$
J_{i n}(k)=-\sum_{j=0}^{N c-1} \sum_{i=1}^{m} u_{i}(k+j)
$$

where $m$ is the number of inputs.

In this paper, we consider only linear constraints on the inputs of the HVLV system

$$
\tilde{u}(k) \geqslant 0
$$

Since the $u(k)$ corresponds to consecutive event occurence times, we have the additional condition

$$
\Delta u(k+j)=u(k+j)-u(k+j-1) \geqslant 0
$$

for $j=1, \ldots, N c$.

Note that the constraint $r(k+j) \geqslant \hat{y}(k+j \mid k)$ for $j=$ $0 \ldots N p-1$ leads to a linear constraint on the inputs.

Theorem A scalar-valued MMPS function can be rewritten into the min-max canonical form

$$
f=\min _{i=1, \ldots, K} \max _{j=1, \ldots, n_{i}}\left(\alpha_{(i, j)}^{T} x+\beta_{(i, j)}\right)
$$

for some integers, $K, n_{1}, \ldots, n_{K}$, vector $\alpha_{(i, j)}$, and real number $\beta_{(i, j)}$.

The objective function of the MPL-MPC problem $J(k)$ can be written in min-max canonical form for MMPS function (미).

\subsection{Illustrative example}

We consider, the HVLV flow shop system presented in the section 2. This system produces two types of products $P 1$ and $P 2$. In developing the control model, a MPS $=(1 \mathrm{P} 1,1 \mathrm{P} 2)$ is given. This model aims to meet the due dates of products subject to JIT production. This would correspond to a scheme in which raw materials are fed to the system as late as possible. Referring to the table $\square$ and the model proposed in section 2, we have the following equations that describe the dynamic of the system:

$$
\begin{gathered}
x_{11}(k)=\max \left(1+x_{11}(k-1), u_{1}(k)\right) \\
x_{22}(k)=\max \left(2+x_{11}(k-1), 2+x_{22}(k-1),\right. \\
\left.1+x_{32}(k-1), 1+u_{1}(k)\right) \\
x_{32}(k)=\max \left(4+x_{11}(k-1), 4+x_{22}(k-1),\right. \\
\left.3+x_{32}(k-1), 3+u_{1}(k), u_{2}(k)\right) \\
y_{1}(k)=2+x_{22}(k) \\
y_{2}(k)=1+x_{32}(k)
\end{gathered}
$$

The MPC approach presented in section 3 is applied to the system and the optimization problem shown in this example is subject to the following constraints:

$$
\begin{aligned}
& 1 \leq \Delta u_{1}(k) \leq 20,1 \leq \Delta u_{2}(k) \leq 20, U(k) \geqslant 0 \\
& \text { Let } N p=N c=2, \lambda=1, X(0)=(0 ; 5 ; 10)
\end{aligned}
$$

Note that $Y(k)$ and $Y(k+1)$ can be expressed as functions of the current state $X(k-1)$ and the future inputs $U(k)$ and $U(k+1)$. Then the MMPS form of the cost criterion is $J(k)=\min \left(\max \left(m_{1}, m_{2}, \ldots, m_{13}, t_{1}\right) ; \max \left(m_{1}, m_{2}\right.\right.$ $\left.\left., \ldots, m_{13}, t_{2}\right) ; \ldots ; \max \left(m_{1}, m_{2}, \ldots, m_{13}, t_{22}\right)\right)$

The optimal MPC strategy for MPL-HVLV system can be computed by solving 22 linear optimization problems and by selecting the overall optimum.

Compute the closed-loop MPC inputs signals over a simulation period $[1,10]$ with references signals $r_{1}(k)$ and $r_{2}(k)$ (see table (1). 
Table 2. Results of simulation

\begin{tabular}{|l|l|l|l|l|l|l|l|l|l|l|}
\hline$k$ & 1 & 2 & 3 & 4 & 5 & 6 & 7 & 8 & 9 & 10 \\
\hline$r_{1}$ & 40 & 44 & 54 & 66 & 75 & 85 & 90 & 100 & 108 & 116 \\
$r_{2}$ & 43 & 50 & 56 & 70 & 80 & 88 & 97 & 106 & 112 & 120 \\
\hline$u_{1}$ & 37 & 41 & 51 & 63 & 72 & 82 & 87 & 97 & 105 & 113 \\
$y_{1}$ & 40 & 44 & 54 & 66 & 75 & 85 & 90 & 100 & 108 & 116 \\
\hline$u_{2}$ & 41 & 49 & 55 & 69 & 79 & 87 & 96 & 105 & 111 & 119 \\
$y_{2}$ & 42 & 50 & 56 & 70 & 80 & 88 & 97 & 106 & 112 & 120 \\
\hline
\end{tabular}

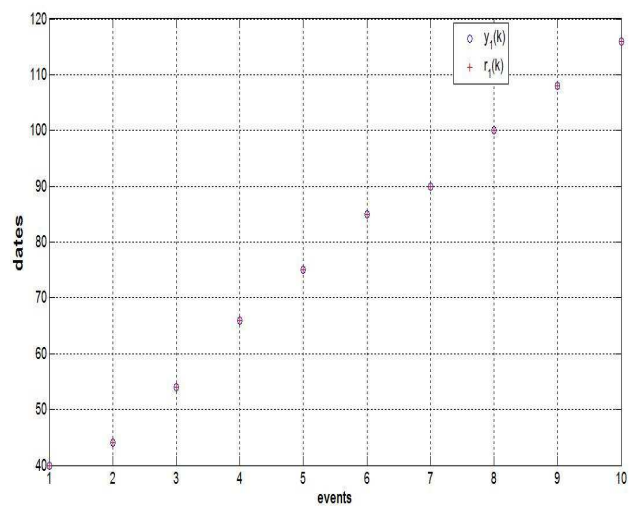

Figure 3. The closed-loop HVLV-MPC output $y_{1}(k)$ and the reference $r_{1}(k)$

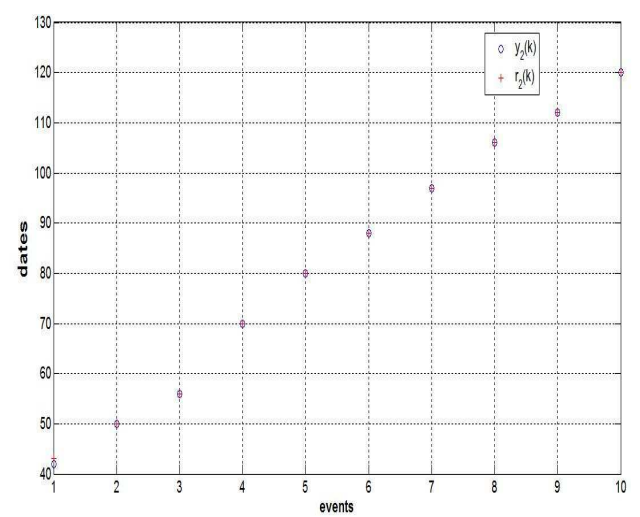

Figure 4. The closed-loop HVLV-MPC output $y_{2}(k)$ and the reference $r_{2}(k)$

\section{Discussion and practical Implementation}

It can be shown that the proposed linear optimization algorithm based on MMPS functions is efficient from tracking reference trajectories point of view. Then, (Table [),(Figure [1]) and (Figure $\mathbf{A}$ ) shows clearly that the output signals are less than or equal to the reference signals. Moreover, (Table D) shows that the input signals respect the different linear constraints mentioned in the above section and they satisfy the JIT production criteria. Then, we can easily see in (Table $\square$ ) that
$1 \leq \Delta u_{1}(k)=u_{1}(k)-u_{1}(k-1) \leq 20,1 \leq \Delta u_{2}(k)=$ $u_{2}(k)-u_{2}(k-1) \leq 20$ and $U(k) \geqslant 0$.

Moreover, for all $1 \leq k \leq 10$, and referring to the above equations for the dynamic of the system, we have: $x_{11}(k)=$ $u_{1}(k)$ and $x_{32}(k)=u_{2}(k)$. This means that the first operation of each product begins as late as possible to meet the due dates.

Using a linear optimization based on MMPS functions don't require a long computation time unlike the nonlinear optimization such as the ELCP approach proposed in [囫 that can become very cumbersome.

\section{Conclusion}

In this work a systematic approach is proposed for direct generation of event-timings for a class of deterministic and decisionfree DEDS systems called HVLV systems. Then, a linear MPC framework is extended from linear discrete-time systems to the HVLV systems to solve a tracking references (due dates) problem.

The popular MPC framework is extended from linear discrete-time systems to the HVLV systems. MPL-MPC based on MMPS functions leads to linear convex optimization problems.

In real-world applications, various uncertainty aspects of the system will perturb its behavior (machines breakdowns, set-up times, urgent orders, etc). In this context, next research work will be done to improve the $(\max ,+)$ model such that it can deal with a dynamic and real time HVLV manufacturing systems scheduling.

\section{References}

[1] F. L. Baccelli, G. Cohen, G. J. Olsder, and J. P. Quadrat. Synchronization and linearity. Wiley New York, 1992.

[2] S. Engell and M. Moser. Two-layer on-line scheduling of flexible manufacturing systems. Proceedings of rensselear second international conference on computer integrated manufacturing, pages 435-443, 1990.

[3] G. Habchi and C. Berchet. A model for manufacturing systems simulation with a control dimension. Simulation Modelling Practice and Theory, 11(1):21 - 44, 2003.

[4] B. D. Schutter and T. J. Boom. MPC for discrete-event systems with soft and hard synchronization constraints. International Journal of Control, 76(1):82-94, 2003.

[5] B. D. Schutter and T. V. D. Boom. Model predictive control for max-plus-linear discrete-event systems: Extended report $\&$ addendum. Delft, Netherlands: Delft University of Technology, 2000.

[6] B. D. Schutter and T. van den Boom. MPC for continuous piecewise-affine systems. Systems \& Control Letters, 52(34): 179 - 192, 2004.

[7] J. W. Seo and T. E. Lee. Steady-state analysis and scheduling of cyclic job shops with overtaking. International Journal of Flexible Manufacturing Systems, 14(4):291-318, 2002.

[8] K. Tamani, R. Boukezzoula, and G. Habchi. Intelligent distributed and supervised flow control methodology for production systems. Engineering Applications of Artificial Intelligence, 22(7):1104 - 1116, 2009.

[9] K. Tamani, R. Boukezzoula, and G. Habchi. Multi-objective supervisory flow control based on fuzzy interval arithmetic: Application for scheduling of manufacturing systems. Simulation Modelling Practice and Theory, 19(5):1371 - 1383, 2011. 